\title{
Microlocal analysis of the scattering angle transform
}

\author{
Christiaan C. Stolk \\ Dept. of Computational \& Applied Mathematics \\ Rice University \\ 6100 Main St. - MS 134 \\ Houston, TX 77005-1892 \\ USA \\ email: cstolk@caam.rice.edu
}

July 23, 2001

\begin{abstract}
The goal of seismic imaging is to map single reflection seismic data to an image of a medium parameter, ie. a reconstruction of the singularities in the medium parameter up to a pseudodifferential factor. Given a smooth model of the medium (background medium), satisfying certain conditions, there is a Fourier integral operator (FIO) mapping seismic data to an image. Under more restrictive conditions the data can be mapped to a family of images, each depending on a different subset of the data. This mapping is used in the determination of the background medium. The canonical relations of these
\end{abstract}


operators consist of data from reflected bicharacteristics in the background medium. When several rays (projections of the bicharacteristics on the base space) connect an acquisition point with a scattering point (multipathing), the conditions for imaging using subsets of data are in general violated. In the geophysical literature scattering angle transforms have been proposed to yield image families in the presence of multipathing. It has been conjectured that an integral operator related to the Kirchhoff migration operator maps seismic data to a family of images. We show that this conjecture is false. The Kirchhoff type angle transform maps seismic data to a sum of a correct image and possible artifacts, ie. singularities in the image that do not correspond to singularities in the medium. We give an explicit example in which such artifacts are present.

\section{Acknowledgements}

The author would like to thank W.W. Symes for valuable discussions. This work was supported in part by The Rice Inversion Project.

\section{Introduction}

Reflection seismic imaging, as currently practiced, is based largely on the linearization of the coefficient-solution relation for the acoustic wave equation (Born approximation, see for instance $[1,2]$ ). The soundspeed function $c$ for example is written as the sum of a smoothly varying background $c_{0}$ ("velocity model") and a small perturbation $\delta c$ that contains the singular- 
ities of $c$. A linear map $F\left[c_{0}\right]$ maps $\delta c$ to an approximation of the data, containing the single reflections. We will refer to this map as the forward map, and we will usually simply write $F$, although $F$ always depends on the background medium.

Geophysicists are particularly interested in the location of discontinuities in the mechanical parameters such as soundspeed, that is in the singular part of $\delta c$. In fact it is often sufficient to reconstruct $\delta c$ up to a pseudodifferential factor $\Psi(x, D)$, that is elliptic on some part of $T^{*} \mathbb{R}^{n} \backslash 0$. We will refer to such a function as an image. To obtain an image an estimate for the background medium $c_{0}$ is needed.

The forward map $F\left[c_{0}\right]$ is an FIO $[3,4,5]$; its canonical relation consists of data for reflected bicharacteristics ("rays"). Consequently, given the background medium $c_{0}$, the singularities of $\delta c$ can be reconstructed from the data under quite general conditions (see $[2,6]$ ). For this problem the data is redundant, since it is a function of $2 n-1$ variables, and $\delta c$ is a function of $n$ variables. Under more restrictive conditions a subset of data can be used to reconstruct the singularities of $\delta c([1,7])$. Subsets that can be used are for instance the data associated with a common source, or with a common value of offset (the difference between source and receiver coordinate). Using such subsets results in a family of images.

The reconstructed $\delta c$ should of course not depend on which subset is used. This semblance principle of migration velocity analysis is used to determine the background medium $c_{0}$ ("velocity analysis"). Construction of image 
families from data subsets is possible when at most a single reflected ray passes over each point in the wavefront set of each data subset [7]. However in general realistic seismic background media are strongly refracting, so that more than one reflected ray pair passes over a portion of the data wave front set. This phenomenon, described precisely below is called multipathing.

In the presence of multipathing reconstruction of $\delta c$ from such a subset is generally no longer possible. Significant artifacts (ie. singularities in the image not present in $\delta c$ ) occur when standard imaging operators are used in this case [7]. When $c_{0}$ is known these artifacts can in principle be removed since their location varies if different subsets of data are used. Formation of an image from the entire data set involves summation of the partial images, during which the artifacts destructively cancel [2]. However, when the correct background velocity is not known it is not possible to distinguish between artifacts and correct image, since the location of both will not be constant. Thus both velocity analysis and image formation are hampered.

So called angle transforms $[8,9,10,11,12]$ have been proposed as a solution to this problem. An angle transform should map the single reflection data to a family of images, each using data associated to a particular value of the scattering angle (in 2 dimensions), or scattering angle and azimuth (in 3 dimensions). The scattering angle is the angle between incoming and outgoing rays at the scattering point, while the azimuth parametrizes the plane orthogonal to the reflector that contains the take off directions of the incoming and outgoing rays. Each image in such a family may depend on 
all the data. In [13] it is shown that there exist Fourier integral operators that map seismic data to such a set of images. To compute such images two methods have been proposed. One is through a Kirchhoff type integral (also called generalized Radon transform) $[8,9,11]$. We will call this type of operator a Kirchhoff type angle transform. The other is using downward continuation of seismic data $[10,12]$.

Although angle transforms have attracted considerable attention in the seismic community, it has not yet been proven that angle transform image families computed in these ways are artifact-free. This question, for the Kirchhoff type angle transform, is the subject of the present paper.

We reach the following conclusions, see Theorem 6.1 and the example in section 7. The composition of the Kirchhoff type angle transform and the forward operator is the sum of a pseudodifferential contribution (which gives a correct image), and a remainder term which may give nonlocal singular contributions, ie. artifacts. We give an explicit example in which such artifacts are present.

It is no easier to resolve correct image and artifact in Kirchhoff type angle transform image families, than in other image decompositions, without knowledge of the correct velocity model $\left(c_{0}\right)$. Therefore the Kirchhoff type angle transform does not provide a good platform for determining $c_{0}$ (velocity analysis): the global semblance principle fails for this way of computing an angle transform.

The structure of the paper is as follows. In section 2 we discuss the 
forward operator $F$. We then quote the result of [2] about imaging using all data in section 3 . In section 4 we introduce a class of operators that includes the Kirchhoff type angle transforms, and the classical imaging operators using subsets of data. We proceed by proving that these operators are Fourier integral operators when composed with a certain pseudodifferential operator that has no effect on the singularities of data in section 5 . In section 6 we show that under certain conditions, applying to the Kirchhoff angle transform, the product of an operator of the class defined in section 4 and the forward operator has a pseudodifferential part. We give equations that have to be satisfied in order for artifacts to be present. Finally in section 7 we discuss an example of a medium with a low velocity lens where such artifacts are present.

A schematic picture of the acquisition geometry in $n=2$ dimensions is given in Figure 1. We assume the medium is in $\mathbb{R}_{+}^{n}$. The acquisition coordinates source position, receiver position and time are denoted by $s, r, t$. We assume they are in a bounded open subset $Y \subset \mathbb{R}_{+}^{2 n-1}(t>0)$. The part of the medium of interest is in some bounded set $X \subset \mathbb{R}_{+}^{n}$. By $x$ we denote the subsurface coordinate, where $x_{n}$ denotes the depth. To indicate that one of these coordinates is a function of some parameters we will sometimes use bold symbols, ie. $\mathbf{r}(x, \alpha)$ is a function associating a receiver coordinate to a subsurface position and take off direction (see the next section).

We will make extensive use of microlocal analysis $[3,14,4,5]$. Covectors are denoted by Greek letters, ie. $(x, \xi) \in T^{*} X \backslash 0,(r, s, t, \rho, \sigma, \tau) \in T^{*} Y \backslash 0$. 


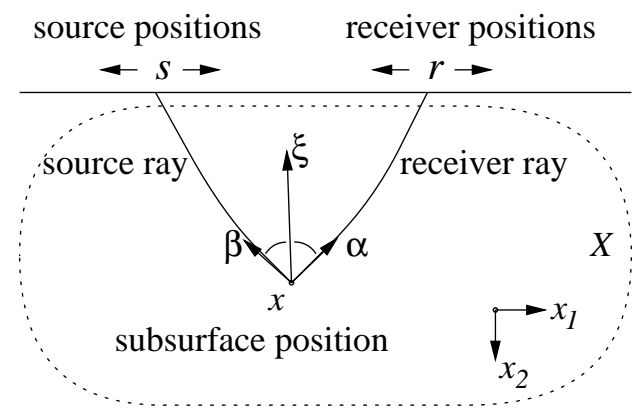

Figure 1: Acquisition geometry in 2 dimensions.

\section{The forward operator}

The forward operator is derived as follows. The pressurefield $u(x, t)$ for a source $f$ in a medium in $\mathbb{R}^{n}$ described by soundspeed $c$ and constant density satisfies the acoustic wave equation

$$
P u:=\left(\frac{1}{c(x)^{2}} \frac{\partial^{2}}{\partial t^{2}}-\sum_{i=1}^{n} \frac{\partial^{2}}{\partial x_{i}^{2}}\right) u(x, t)=f(x, t) .
$$

The causal Green's function $G(x, s, t)$ is given by the solution when $f(x, t)=$ $\delta(x-s) \delta(t)$ under the condition that $G=0$ when $t<0$ (here $s \in \mathbb{R}^{n}$ ).

To obtain an approximation for the scattered field a formal linearization in $c$ is done, by writing $c(x)=c_{0}(x)+\delta c(x)$. It is assumed that $c_{0} \in C^{\infty}\left(\mathbb{R}^{n}\right)$. The perturbation of $P$ is given by $\delta P=-\frac{2 \delta c(x)}{c_{0}(x)^{3}} \frac{\partial^{2}}{\partial t^{2}}$. The perturbation of the Green's function satisfies the equation

$$
P \delta G=-\delta P G
$$

It follows that

$$
\delta G(x, s, t)=\int \mathrm{d} x_{0} \mathrm{~d} t_{0} G\left(x, x_{0}, t-t_{0}\right) \frac{2 \delta c\left(x_{0}\right)}{c\left(x_{0}\right)^{3}} \frac{\partial^{2}}{\partial t^{2}} G\left(x_{0}, s, t_{0}\right) .
$$


The (idealized) seismic data is given by $\delta G(r, s, t),(r, s, t) \in Y$. The forward operator is given by the map

$$
F\left[c_{0}\right]: \delta c \mapsto \delta G,
$$

where $\delta G$ is seen as a function of $(r, s, t) \in Y$. In this derivation it is assumed that the acquisition surface is embedded in the medium, ie. it is ignored that in fact acquisition takes place on the boundary of the medium. In practice the effects of the boundary (such as surface waves and reflections at the boundary) can be suppressed.

It has been shown in $[15,2]$ that under certain conditions the forward operator $F$ mapping $\delta c$ to data is a Fourier integral operator (FIO, see references $[3,4,5]$ for their definition and properties). The conditions are the absence of direct rays from source to receiver and the absence of so called grazing rays, rays that are tangent to the acquisition surface. In fact this is true microlocally. The composition of the forward operator with a pseudodifferential cutoff that mutes data microlocally in a neighborhood of direct rays and grazing rays is a FIO.

In the remainder the operator $F$ will be defined including such a pseudodifferential cutoff. In addition there will be a cutoff such that $F(r, s, t, x)=0$ for $(r, s, t)$ in a neighborhood of the boundary of $Y$. By choosing $X$ sufficiently large, we may assume that the kernel of $F$ vanishes for $x$ in a neighborhood of the boundary of $X$, because $Y$ is bounded and for given $(r, s, t) \in Y$ the kernel $F(r, s, t, x)$ is nonzero only on a bounded set of $x$ by the finite propagation speed of waves. Defined in this way the operator $F$ 
maps $\mathcal{D}^{\prime}(X)$ to $\mathcal{D}^{\prime}(Y)$.

To give an expression for the canonical relation we first introduce some notation. The bicharacteristics are described by a Hamiltonian system, that can be reduced to a system of dimension $2 n-1$ for $(x, \alpha)$, where $\alpha \in$ $S^{n-1}$ is the unit vector pointing in the direction of propagation $[16,3]$. If $\xi, \tau$ denote the covectors corresponding to $x, t$, then $\alpha=\frac{-\tau^{-1} \xi}{\left\|\tau^{-1} \xi\right\|}$. Let $\mathbf{x}\left(x_{0}, \alpha_{0}, t\right), \boldsymbol{\alpha}\left(x_{0}, \alpha_{0}, t\right)$ be the solution to this system with initial values $\left(x_{0}, \alpha_{0}\right)$ at time $t=0$. By $x$ we will denote the scattering point, and by $\beta$ the take off direction of the source ray from $x$. When this ray hits the acquisition surface nontangently, then the time $t$ can locally be solved from the equation $\mathbf{x}_{n}(x, \beta, t)=0$. We will assume that each ray intersects the acquisition surface at most once. The solution for $t$ will be denoted by $\phi_{s}(x, \beta)$. The source coordinate and slowness at this time will be denoted by

$$
\begin{aligned}
\mathbf{s}(x, \beta) & =\left(\mathbf{x}_{1}\left(x, \beta, \phi_{s}(x, \beta)\right), \ldots, \mathbf{x}_{n-1}\left(x, \beta, \phi_{s}(x, \beta)\right)\right), \\
\mathbf{p}_{s}(x, \beta) & =c_{0}(s)^{-1}\left(\boldsymbol{\alpha}_{1}\left(x, \beta, \phi_{s}(x, \beta)\right), \ldots, \boldsymbol{\alpha}_{n-1}\left(x, \beta, \phi_{s}(x, \beta)\right)\right),
\end{aligned}
$$

where $\alpha_{i}$ denotes the $i$-th component of the unit vector $\alpha$. In the same way we define $\phi_{r}(x, \alpha), \mathbf{r}(x, \alpha), \mathbf{p}_{r}(x, \alpha)$. The canonical relation is given by

$$
\begin{aligned}
\Lambda= & \left\{\left(\mathbf{r}(x, \alpha), \mathbf{s}(x, \beta), \phi_{r}(x, \alpha)+\phi_{s}(x, \beta),-\tau \mathbf{p}_{r}(x, \alpha),-\tau \mathbf{p}_{s}(x, \beta), \tau\right.\right. \\
& \left.\left.x, \frac{-\tau}{c_{0}(x)}(\alpha+\beta)\right) \mid(x, \alpha, \beta) \in K, \tau \in \mathbb{R} \backslash 0\right\}
\end{aligned}
$$

where $K \subset \mathbb{R}^{n} \times\left\{(\alpha, \beta) \in S^{n-1} \times S^{n-1} \mid \alpha+\beta \neq 0\right\}$. The set $K$ contains take off positions and angles for rays hitting the acquisition surface (excluding 
open sets around grazing rays and around $\alpha+\beta=0$ ). See also Figure 1.

Since $F$ is a FIO, and because of the cutoffs included in its definition, its distribution kernel can be written as a finite sum of expressions of the form

$$
\int \mathrm{d} \theta^{(i)} A^{(i)}\left(y, x, \theta^{(i)}\right) \mathrm{e}^{\mathrm{i} \phi^{(i)}\left(y, x, \theta^{(i)}\right)}
$$

see $[3,4]$. Here $\theta^{(i)}$ is in $\mathbb{R}^{N^{(i)}}$ for some suitable $N^{(i)} \geq 1, \phi^{(i)}: Y \times X \times$ $\mathbb{R}^{N^{(i)}} \supset \rightarrow \mathbb{R}$ is a nondegenerate phase function, and $A^{(i)}: Y \times X \times \mathbb{R}^{N^{(i)}} \supset \rightarrow$ $\mathbb{R}$ is an amplitude which is in some symbol class. The phase functions can in general be chosen in many ways, which in turn leads to different expressions for the amplitude.

The local expressions (3) each describe the operator on a subset of $\Lambda$. For the actual evaluation of such integrals the number of $\theta^{(i)}$ integrations should be as low as possible. To describe the operator on a neighborhood of some point of $\Lambda$ given by $(x, \alpha, \beta, \tau)$ the minimum number of phase variables is given by the corank of the projection $T \pi: T \Lambda \rightarrow T(Y \times X)$ at $(x, \alpha, \beta, \tau)$. In this case

$$
\operatorname{corank} T \pi=1+\operatorname{corank} \frac{\partial r}{\partial \alpha}(x, \alpha)+\operatorname{corank} \frac{\partial s}{\partial \beta}(x, \beta) .
$$

The corank $>1$ precisely when $s$ or $r$ are in a caustic point relative to $x$.

Let

$$
\tilde{\Lambda}=\Lambda \backslash(\text { a closed neighborhood of }\{\lambda \in \Lambda, \operatorname{corank} T \pi(\lambda)>1\}) .
$$

This part of the canonical relation can be described by phase functions of the form $\tau\left(t-T^{(i)}(x, r, s)\right)$, where $T^{(i)}$ is the two way traveltime from $s$ to 
$x$ to $r$, and $(x, r, s)$ in some suitable domain $D^{(i)}$. In other words, there are $\Lambda^{(i)}, i \in I$, each given by

$$
\begin{aligned}
\Lambda^{(i)}= & \left\{\left(r, s, T^{(i)}(x, r, s),-\tau \frac{\partial T^{(i)}}{\partial r}(x, r, s),-\tau \frac{\partial T^{(i)}}{\partial s}(x, r, s), \tau ;\right.\right. \\
& \left.\left.x, \tau \frac{\partial T^{(i)}}{\partial x}(x, r, s)\right) \mid(x, r, s) \in D^{(i)}, \tau \in \mathbb{R} \backslash 0\right\},
\end{aligned}
$$

such that $\bigcup_{i \in I} \Lambda^{(i)}=\Lambda$. We write $K^{(i)}$ for the subset of $K$ associated with $\Lambda^{(i)}$. The part $\tilde{F}$ of $F$ associated with $\tilde{\Lambda}$ can therefore be written in the form

$$
\sum_{i \in I} \int \mathrm{d} \tau A^{(i)}(r, s, t, x, \tau) \mathrm{e}^{\mathrm{i} \tau\left(t-T^{(i)}(x, r, s)\right)}
$$

where $A^{(i)}(x, r, s, t, \tau), i \in I$ is a suitable set of amplitude functions. By $F^{(i)}$ we will denote the $i$-th contribution in (5).

\section{$3 \quad$ Imaging using all the data}

It is our goal to reconstruct the position of the singularities in $\delta c$, given the background medium $c_{0}$. With this in mind we say an operator $H$ is an imaging operator (produces a correct image), when

$$
H \circ F=(\psi \text { do }) \text {. }
$$

This $\psi$ do should be elliptic at least microlocally in some part of $T^{*} X \backslash 0$. Due to the geometry of the rays and the acquisition geometry in general not all $(x, \xi) \in T^{*} X \backslash 0$ can be "illuminated" by rays from the surface, so this is all one can expect. 
By an artifact we mean a singularity in the image, where there is no singularity in $\delta c$. Artifacts can occur when $H \circ F$ is not pseudodifferential. The presence of artifacts is only determined by the kinematics (the Lagrangian manifold, the phase functions). The precise value of the amplitudes is therefore generally not important in this paper.

We first quote a result about imaging using all the data. It has been shown in [2] that the adjoint operator $F^{*}$ is an imaging operator under the so called traveltime injectivity condition (TIC).

Definition 1 We say the background medium $c_{0}$ satisfies $T I C$ if the projection

$$
\pi_{Y}: \Lambda \rightarrow T^{*} Y \backslash 0
$$

is an embedding.

This is slightly different from the definition in [2], where it was just required that $\pi_{Y}$ is injective. Here in addition $\pi_{Y}$ has to be proper (since it is an immersion, see [2]). This can be obtained by applying a pseudodifferential cutoff operator to the data so that the cutoff data is smooth microlocally on a neighborhood of the set where TIC is violated, see [6]. Under this condition the composition $F^{*} F$ is a pseudodifferential operator.

If $F$ is given by a sum of contributions (3), then the operator $F^{*}$ is a sum of local Fourier integral operators with phase function $-\phi^{(i)}$ and amplitude $\overline{A^{(i)}}$. In practice it is often sufficient to use only the part of the operator associated with $\tilde{\Lambda}$, where $\operatorname{corank} T \pi=1$. Therefore in this paper we use only 
the part of $F$ given by (5) for imaging. This leads to an imaging operator with the following kernel

$$
\sum_{i \in I} \int \mathrm{d} \tau \overline{A^{(i)}(x, r, s, t, \tau)} e^{\mathrm{i} \tau\left(T^{(i)}(x, r, s)-t\right)} .
$$

\section{A class of operators}

The operators that we will study can be described in the following way. Let $\mathbf{e}=\mathbf{e}(x, \alpha, \beta)$ be a smooth function on the canonical relation $\Lambda$, that takes its values in some set $E \subset \mathbb{R}^{n-1}$, and is homogeneous of order 0 in the conic variables. On the subsets $\Lambda^{(i)}$, given above (5), this leads to functions

$$
\mathbf{e}^{(i)}:(x, r, s) \mapsto \mathbf{e}^{(i)}(x, r, s)
$$

Given such a function e the distribution kernel of the operators that we will study is defined by

$$
\begin{aligned}
H(x, e ; r, s, t) & =\sum_{i \in I} F^{(i)^{*}}(x, r, s, t) \delta\left(e-\mathbf{e}^{(i)}(x, r, s)\right) \\
& =\sum_{i \in I} \int \mathrm{d} \tau \overline{A^{(i)}(x, r, s, t, \tau)} \delta\left(e-\mathbf{e}^{(i)}(x, r, s)\right) \mathrm{e}^{\mathrm{i} \tau\left(T^{(i)}(x, r, s)-t\right)} .
\end{aligned}
$$

Using Theorem 1.3.6 in [3] it can be shown that the product of distributions in the first line of (7) is a well defined distribution. Therefore this operator maps $C^{\infty}$ functions on $Y$ to $C^{\infty}$ functions on $X \times E$, which can be viewed as a family of functions on $X$, indexed by $e \in E$.

Since we are mainly interested in the position of the singularities in the image, the precise value of the amplitude is not relevant here. The amplitude 
should be $C^{\infty}$ and its support should be contained in the support of $T^{(i)}$ and in the support of the data. Moreover it is convenient when the phase of the amplitude is chosen as in (7).

The classical Kirchhoff imaging formulas using common source data, or common offset data are obtained by choosing $e$ equal to the source coordinate,

$$
\mathbf{e}(x, \alpha, \beta)=\mathbf{s}(x, \beta)
$$

or to the offset coordinate

$$
\mathbf{e}(x, \alpha, \beta)=\mathbf{r}(x, \alpha)-\mathbf{s}(x, \beta)
$$

In this case for each $x$ the same set of data is used to produce the image. Our definition in fact allows to use a different subset of data for each subsurface point $(r, s)$.

The Kirchhoff type scattering angle transform, as proposed in $[8,9,11]$ is obtained in the following way. For $n=2$ dimensions $\mathbf{e}$ is defined to be the scattering angle. Let $\tilde{\alpha}, \tilde{\beta} \in \mathbb{R}$ be such that $\alpha=(\cos \tilde{\alpha}, \sin \tilde{\alpha}), \beta=$ $(\cos \tilde{\beta}, \sin \tilde{\beta})$. Then we define the (signed) scattering angle as

$$
\mathbf{e}(x, \alpha, \beta)=\tilde{\alpha}-\tilde{\beta} .
$$

Of course only its value modulo $2 \pi$ is relevant, so we may require $-\pi<$ $\mathbf{e}(x, \alpha, \beta) \leq \pi$. This leads to a similar formula as was studied in [8]. For $n>2$ dimensions $\mathbf{e}$ is chosen to be scattering angle and azimuth, which can 
be defined by

$$
\mathbf{e}(x, \alpha, \beta)=\left(\arccos (\alpha \cdot \beta), \frac{1}{2 \sin (\arccos (\alpha \cdot \beta) / 2)}(-\alpha+\beta) \in(0, \pi) \times S^{n-2},\right.
$$

see [17]. Here $E$ is the manifold $(0, \pi) \times S^{n-2}$. We assume that the image of e can be parametrized by a subset of $\mathbb{R}^{n-1}$. The operator $H$ for this case was studied (for elastic media) in $[9,11]$.

Let $\nu=\frac{\alpha+\beta}{\|\alpha+\beta\|}$. In these cases $(x, \nu, e, \tau)$ forms a set of coordinates on $\Lambda$. The map e in (11) has a singularity at $\alpha=\beta$. (In principle, by choosing $\mathbf{e}$ differently, this could be avoided while keeping the property that $(x, \nu, e, \tau)$ forms a set of coordinates on $\Lambda$.)

In all these examples an image using all the data (as discussed in section 3) is obtained by integration over $e$.

\section{$5 \quad$ Fourier integral operators}

In this section we are interested in the question whether an operator $H$ of the type defined in the previous section is a Fourier integral operator. Let $H^{(i)}$ be defined by taking the $i$-th contribution in the sum in (7). The kernel of $H^{(i)}$ can be written as an oscillatory integral by expanding the $\delta$-function

$$
\begin{aligned}
H^{(i)}(x, e, r, s, t)= & \frac{1}{(2 \pi)^{n-1}} \int \mathrm{d} \tau \mathrm{d} \epsilon \overline{A^{(i)}(x, r, s, t, \tau)} \\
& \times e^{\mathrm{i} \tau\left(T^{(i)}(x, r, s)-t\right)+\mathrm{i}\left\langle\epsilon, e-\mathbf{e}^{(i)}(x, r, s)\right\rangle} .
\end{aligned}
$$

Its looks like $H^{(i)}$ may be a Fourier integral operator with frequency variables $(\epsilon, \tau)$ and phase function $\tau\left(T^{(i)}(x, r, s)-t\right)+\left\langle\epsilon, e-\mathbf{e}^{(i)}(x, r, s)\right\rangle$. This is not 
the case, because the amplitude function $\overline{A^{(i)}(x, r, s, t, \tau)}$ does in general not have the correct decay of derivatives on a conic neighborhood of $\tau=0$, $\epsilon \neq 0$.

However, we will show that the composition of $H$ and a suitable cutoff $\psi\left(D_{r}, D_{s}, D_{t}\right)$ in frequency space is a Fourier integral operator. Let $\psi(\rho, \sigma, \tau)=0$ on a closed conic neighborhood $K$ of $\{(\rho, \sigma, \tau) \mid \tau=0,(\rho, \sigma) \neq$ $0\}$, and let $\psi(\rho, \sigma, \tau)=1$ outside a small conic neighborhood of $K$. Data is not affected by such a pseudodifferential operator in the sense that the compose of $\psi\left(D_{r}, D_{s}, D_{t}\right)$ and $F$ is equal to $F$ up to a regularizing operator (the projection of $\Lambda$ on $T^{*} Y \backslash 0$ is in the elliptic region of $\psi\left(D_{r}, D_{s}, D_{t}\right)$ ). We will use the following notation

$$
\begin{aligned}
& \boldsymbol{\xi}^{(i)}(x, r, s, \tau, \epsilon)=-\left\langle\epsilon, \frac{\partial \mathbf{e}^{(i)}}{\partial x}(x, r, s)\right\rangle+\tau \frac{\partial T^{(i)}}{\partial x}(x, r, s), \\
& \boldsymbol{\rho}^{(i)}(x, r, s, \tau, \epsilon)=\left\langle\epsilon, \frac{\partial \mathbf{e}^{(i)}}{\partial r}\right\rangle-\tau \frac{\partial T^{(i)}}{\partial r} \\
& \boldsymbol{\sigma}^{(i)}(x, r, s, \tau, \epsilon)=\left\langle\epsilon, \frac{\partial \mathbf{e}^{(i)}}{\partial s}\right\rangle-\tau \frac{\partial T^{(i)}}{\partial s}
\end{aligned}
$$

Theorem 5.1 The composition $H \circ \psi\left(D_{r}, D_{s}, D_{t}\right)$ is a Fourier integral operator with canonical relation given by

$$
\begin{aligned}
C= & \bigcup_{i \in I}\left\{\left(x, \mathbf{e}^{(i)}(x, r, s), \boldsymbol{\xi}^{(i)}(x, r, s, \tau, \epsilon), \epsilon ; r, s, T^{(i)}(x, r, s), \boldsymbol{\rho}^{(i)}(x, r, s, \tau, \epsilon)\right.\right. \\
& \left.\left.\boldsymbol{\sigma}^{(i)}(x, r, s, \tau, \epsilon), \tau\right) \mid(x, r, s) \in D^{(i)}, \epsilon \in \mathbb{R}^{n-1}, \tau \in \mathbb{R} \backslash 0\right\}
\end{aligned}
$$

The operator $H$ maps $\mathcal{D}^{\prime}(Y)$ to $\mathcal{D}^{\prime}(X \times E)$. 
Proof Let $u$ be in $\mathcal{D}^{\prime}(Y)$. We study the map $u \mapsto H^{(i)} u$. Consider first the function

$$
f_{1}(x, r, s, t):=\int \mathrm{d} \tau \mathrm{d} \tilde{t} \overline{A^{(i)}(x, r, s, \tilde{t}, \tau)} \mathrm{e}^{\mathrm{i} \tau(t-\tilde{t})}\left(\psi\left(D_{r}, D_{s}, D_{t}\right) u\right)(r, s, \tilde{t})
$$

This can be written for some modified amplitude $B(x, r, s, \tau)$ independent of the time variable $\tilde{t}$ as

$$
\begin{aligned}
& \int \mathrm{d} \tau \mathrm{d} \tilde{t} B(x, r, s, \tau) \mathrm{e}^{\mathrm{i} \tau(t-\tilde{t})}\left(\psi\left(D_{r}, D_{s}, D_{t}\right) u\right)(r, s, \tilde{t}) \\
& \quad+R_{1}(x, r, s)\left(\psi\left(D_{r}, D_{s}, D_{t}\right) u\right),
\end{aligned}
$$

where $R_{1}(x, r, s)$ is an operator that is regularizing in the time variable. Since $\psi\left(D_{r}, D_{s}, D_{t}\right) u$ is smooth microlocally around $\tau=0,(\rho, \sigma) \neq 0$, it follows that $R_{1}(x, r, s)\left(\psi\left(D_{r}, D_{s}, D_{t}\right) u\right)$ is a smooth function of $(x, r, s, t)$. The composition $R_{2}:=R_{1} \circ \psi\left(D_{r}, D_{s}, D_{t}\right)$ is regularizing in $(r, s, t)$.

Writing out the action of $\psi\left(D_{r}, D_{s}, D_{t}\right)$ on $u$ explicitly we find that

$$
\begin{aligned}
f_{1}(x, r, s, t)= & \frac{1}{(2 \pi)^{2 n-2}} \int \mathrm{d}(\rho, \sigma, \tau, \tilde{r}, \tilde{s}, \tilde{t}) B(x, r, s, \tau) \psi(\rho, \sigma, \tau) \\
& \times \mathrm{e}^{\mathrm{i}(\tau(t-\tilde{t})+\langle\rho, r-\tilde{r}\rangle+\langle\sigma, s-\tilde{s}\rangle)} u(\tilde{r}, \tilde{s}, \tilde{t})+\left(R_{2} u\right)(x, r, s, t) .
\end{aligned}
$$

Hence the map $u \mapsto f_{1}$ is a Fourier integral operator with canonical relation

$$
\begin{aligned}
\left\{(x, r, s, t, 0, \rho, \sigma, \tau ; r, s, t, \rho, \sigma, \tau) \mid(x, r, s) \in D^{(i)}, t \in \mathbb{R},\right. & \\
& \left.(\rho, \sigma) \in \mathbb{R}^{2 n-2}, \tau \in \mathbb{R} \backslash 0\right\} .
\end{aligned}
$$

The result of applying $H^{(i)} \circ \psi\left(D_{r}, D_{s}, D_{t}\right)$ to $u$ can be written as the following integral of $f_{1}$

$$
\left(H^{(i)} u\right)(x, e)=\int \mathrm{d} r \mathrm{~d} s \mathrm{~d} t \delta\left(t-T^{(i)}(x, r, s)\right) \delta\left(e-\mathbf{e}^{(i)}(x, r, s)\right) f_{1}(x, r, s, t),
$$


(this integral is well defined if $u$ is smooth). In this expression the $\delta$-functions can be expanded as in (12). It follows from this expansion that the map $f_{1} \mapsto H^{(i)} u$ is a Fourier integral operator with canonical relation given by

$$
\begin{aligned}
& \left\{\left(x, \mathbf{e}^{(i)}, \xi, \epsilon ; x, r, s, T^{(i)}, \xi+\left\langle\epsilon, \frac{\partial \mathbf{e}^{(i)}}{\partial x}\right\rangle-\tau \frac{\partial T^{(i)}}{\partial x},\left\langle\epsilon, \frac{\partial \mathbf{e}^{(i)}}{\partial r}\right\rangle-\tau \frac{\partial T^{(i)}}{\partial r}\right.\right. \\
& \left.\left.\left\langle\epsilon, \frac{\partial \mathbf{e}^{(i)}}{\partial s}\right\rangle-\tau \frac{\partial T^{(i)}}{\partial s}, \tau\right) \mid(x, r, s) \in D^{(i)},(\xi, \epsilon, \tau) \in \mathbb{R}^{2 n} \backslash 0\right\} .
\end{aligned}
$$

Here we omitted the argument $(x, r, s)$ of the functions $T^{(i)}, \mathbf{e}^{(i)}$.

The map $H^{(i)} \circ \psi\left(D_{r}, D_{s}, D_{t}\right)$ is hence the compose of two local Fourier integral operators. The calculus of local Fourier integral operators can now be applied, see Theorem 2.4.1 in [3]. Indeed the conditions of this theorem are satisfied, and the composition is a FIO with canonical relation given by the $i$-the contribution in the union (13). This proves the first part of the theorem.

For the proof of the second statement note that $\frac{\partial T^{(i)}}{\partial x}(x, r, s) \neq 0$. Therefore $\left(\boldsymbol{\xi}^{(i)}(x, r, s, \tau, \epsilon), \epsilon\right)=0$ implies that $\left(\boldsymbol{\rho}^{(i)}(x, r, s, \tau, \epsilon), \boldsymbol{\sigma}^{(i)}(x, r, s, \tau, \epsilon), \tau\right)$ $=0$. It follows that the canonical relation contains no elements of the form $(x, e, 0,0 ; r, s, t, \rho, \sigma, \tau)$. Furthermore the operator $H$ is a finite sum of local FIO's with bounded support. This implies the statement. 


\section{Composition of imaging operator and forward operator: image and artifacts}

In this section we study the singular part of the composition $H \circ F$ of an operator $H$ of the type described in section 4 and the forward operator $F$. We assume both have the same background medium $c_{0}$. We will give two theorems about such operators, and then assess whether the conditions are satisfied for the examples of section 4. This is the product of two Fourier integral operators (the pseudodifferential operator $\psi$ can be omitted, since $\psi F=F$ modulo a regularizing operator).

The compose $H \circ F$ is a well defined operator $\mathcal{D}^{\prime}(X) \rightarrow \mathcal{D}^{\prime}(X \times E)$. Its wave front set is contained in the composition of the wave front sets of

$H$ and $F$ (see [3], Theorem 1.3.7), hence in the composition of canonical relation $C \circ \Lambda$, which is defined by

$$
\begin{aligned}
C \circ \Lambda= & \left\{\left(x_{1}, e, \xi_{1}, \epsilon ; x_{2}, \xi_{2}\right) \mid \text { there is }(r, s, t, \rho, \sigma, \tau)\right. \text { with } \\
& \left.\left(x_{1}, e, \xi_{1}, \epsilon ; r, s, t, \rho, \sigma, \tau\right) \in C \text { and }\left(r, s, t, \rho, \sigma, \tau ; x_{2}, \xi_{2}\right) \in \Lambda\right\} .
\end{aligned}
$$

This leads to the following equations

$$
\begin{aligned}
r & =\mathbf{r}(x, \alpha) \\
s & =\mathbf{s}(x, \beta) \\
T^{(i)}(\bar{x}, r, s) & =\phi_{r}(x, \alpha)+\phi_{s}(x, \beta) \\
\boldsymbol{\rho}^{(i)}(\bar{x}, r, s, \tau, \epsilon) & =-\tau \mathbf{p}_{r}(x, \alpha) \\
\boldsymbol{\sigma}^{(i)}(\bar{x}, r, s, \tau, \epsilon) & =-\tau \mathbf{p}_{s}(x, \beta)
\end{aligned}
$$


and we have

$$
\begin{aligned}
C \circ \Lambda= & \bigcup_{i \in I}\left\{\left(\bar{x}, \mathbf{e}^{(i)}(\bar{x}, r, s), \boldsymbol{\xi}^{(i)}(\bar{x}, r, s, \tau, \epsilon), \epsilon ; x, \frac{-\tau}{c_{0}(x)}(\alpha+\beta) \mid\right.\right. \\
& (\bar{x}, r, s, \tau, \epsilon, x, \alpha, \beta) \text { satisfy }(14)\} .
\end{aligned}
$$

The equations (14) determine the positions of possible singularities in the image. Because the projection of $C \cap\{\epsilon=0\}$ along the $e$-axis is equal to $\tilde{\Lambda},(14)$ has for each $i$ a set of solutions given by

$$
\begin{array}{r}
\{(\bar{x}, r, s, \tau, \epsilon, x, \alpha, \beta) \mid r=\mathbf{r}(x, \alpha), s=\mathbf{s}(x, \beta), \bar{x}=x, \epsilon=0 \\
\left.(x, \alpha, \beta) \in K^{(i)}, \tau \in \mathbb{R} \backslash 0\right\}
\end{array}
$$

These solutions yield the correct image. In the following theorem we address the question whether the singular contributions to $H \circ F$ from these solutions are pseudodifferential. In addition (14) may have different solutions, leading to artifacts. An example is given in section 7 below. When $\epsilon \neq 0$ in the wave front set of such contributions, they can in principle be removed by applying a cutoff in the Fourier domain.

We define $\Psi$ to be the map from $\Lambda$ to $T^{*} X \backslash 0 \times E$ given by

$$
\Psi(x, \alpha, \beta, \tau)=\left(x, \frac{-\tau}{c_{0}(x)}(\alpha+\beta), \mathbf{e}(x, \alpha, \beta)\right)
$$

By $\Psi^{(i)}(x, r, s, \tau)$ we will denote a local representation on $\Lambda^{(i)}$.

Theorem 6.1 Assume that the traveltime injectivity condition (TIC) holds. Assume that for each $i$ the map $\Psi^{(i)}$ is locally diffeomorphic. Then the 
composition $H \circ F$ is given by the sum of a smooth family of pseudodifferential operators parametrized by $e$ and an operator $R$

$$
H \circ F=W\left(x, e, D_{x}\right)+R,
$$

where $R$ is in general not pseudodifferential and the projection of the wave front set of $R$ on the $(x, e, \xi, \epsilon)$ variable contains no elements with $\epsilon=0$.

Denote by $C^{(i)}$ be the $i$-th part in the union (13).

Lemma 6.2 The canonical relation $C^{(i)}$ corresponds locally to a diffeomorphism $T^{*} Y \backslash 0 \rightarrow T^{*}(X \times E) \backslash 0$ if and only if

$$
\frac{\partial\left(T^{(i)}, \boldsymbol{\rho}^{(i)}, \boldsymbol{\sigma}^{(i)}\right)}{\partial(x, \epsilon)}
$$

is invertible. When $\epsilon=0$ a necessary and sufficient condition is that the map $\Psi^{(i)}$ is locally diffeomorphic.

Proof To show that the canonical relation $C^{(i)}$ corresponds locally to a diffeomorphism $T^{*} Y \backslash 0 \rightarrow T^{*}(X \times E) \backslash 0$ it is sufficient to show that the projection of $C^{(i)}$ on one of the sets $T^{*} Y \backslash 0$ or $T^{*}(X \times E) \backslash 0$ is a local diffeomorphism, see [4], lemma 25.3.6. Coordinates for $C^{(i)}$ are given by $(x, r, s, \tau, \epsilon)$. The map $(x, r, s, \tau, \epsilon) \mapsto(r, s, t, \rho, \sigma, \tau)$ is a local diffeomorphism if and only if (17) has maximal rank, this proves the first statement.

When $\epsilon=0$ the matrix (17) can be written as

$$
\left.\frac{\partial\left(T^{(i)}, \boldsymbol{\rho}^{(i)}, \boldsymbol{\sigma}^{(i)}\right)}{\partial(x, \epsilon)}\right|_{\epsilon=0}=\left(\begin{array}{cc}
\frac{\partial T^{(i)}}{\partial x} & 0 \\
-\tau \frac{\partial^{2} T^{(i)}}{\partial r \partial x} & \left(\frac{\partial \mathbf{e}^{(i)}}{\partial r}\right)^{\mathrm{t}} \\
-\tau \frac{\partial^{2} T^{(i)}}{\partial s \partial x} & \left(\frac{\partial \mathbf{e}^{(i)}}{\partial s}\right)^{\mathrm{t}}
\end{array}\right)=\left(\begin{array}{ccc}
\frac{\partial \boldsymbol{\xi}^{(i)}}{\partial \tau} & \frac{\partial \boldsymbol{\xi}^{(i)}}{\partial r} & \frac{\partial \boldsymbol{\xi}^{(i)}}{\partial s} \\
0 & \frac{\partial \mathbf{e}^{(i)}}{\partial r} & \frac{\partial \mathbf{e}^{(i)}}{\partial s}
\end{array}\right)^{\mathrm{t}} .
$$


The map $\Psi^{(i)}$ is a local diffeomorphism precisely when the matrix on the right hand side is nonsingular.

Proof of Theorem 6.1 The projection of $C \cap\{\epsilon=0\}$ along the $e$-axis is equal to the set $\tilde{\Lambda}$. By the traveltime injectivity condition it follows that there is a neighborhood $U$ of $C \cap\{\epsilon=0\}$, such that all elements of $U \circ \Lambda$ correspond to solutions of the type (16). Let $H_{U}$ be a Fourier integral operator equal to $H$ on a neighborhood of $C \cap\{\epsilon=0\}$, with canonical relation $U$. The wave front set of $H-H_{U}$ contains no elements with $\epsilon=0$.

To complete the proof we show that $H_{U} \circ F$ is a Fourier integral operator with canonical relation contained in

$$
\left\{(x, \xi, e, 0 ; x, \xi) \mid(x, \xi) \in T^{*} X \backslash 0, e \in E\right\}
$$

The calculus of Fourier integral operators (see e.g. [3], theorem 2.4.1) gives sufficient conditions that the composition of two the FIO's $H_{U}$ and $F$ is again a FIO. The essential condition is that the composition of canonical relations is transversal, meaning that $L=U \times \Lambda$ and $M=T^{*}(X \times E) \backslash 0 \times$ $\operatorname{diag} T^{*} Y \backslash 0 \times T^{*} X \backslash 0$ intersect transversally.

In a neighborhood of a point in $\Lambda$ given by (16), $\Lambda$ can be parametrized by (4). Using this parametrization one finds that the composition is transversal if and only if the matrix

$$
\frac{\partial}{\partial(\bar{x}, x, \epsilon)}\left(\begin{array}{c}
T^{(i)}(\bar{x}, r, s)-T^{(i)}(x, r, s) \\
\boldsymbol{\rho}^{(i)}(\bar{x}, r, s, \tau, \epsilon)-\boldsymbol{\rho}^{(i)}(x, r, s, \tau, 0) \\
\boldsymbol{\sigma}^{(i)}(\bar{x}, r, s, \tau, \epsilon)-\boldsymbol{\sigma}^{(i)}(x, r, s, \tau, 0)
\end{array}\right)
$$


has maximal rank when $\bar{x}=x, \epsilon=0$. Therefore a sufficient condition is that any of the conditions in the Lemma 6.2 are satisfied. Since at $\bar{x}=x, \epsilon=0$ the $x$ derivatives are not linearly independent from the $\bar{x}$ derivatives the condition that $\Psi^{(i)}$ is locally diffeomorphic is necessary and sufficient for the intersection to be transversal.

The other conditions of theorem 2.4.1 in [3] are satisfied as well. It follows that $H_{U} \circ F$ is a Fourier integral operator with canonical relation

$$
\begin{aligned}
& \bigcup_{i \in I}\left\{\left(x, \boldsymbol{\xi}^{(i)}(x, r, s, \tau, 0), \mathbf{e}^{(i)}(x, r, s, \tau, 0), 0 ; x, \boldsymbol{\xi}^{(i)}(x, r, s, \tau, 0) \mid\right.\right. \\
& \left.\quad(x, r, s) \in D^{(i)}, \tau \in \mathbb{R} \backslash 0\right\} .
\end{aligned}
$$

This proves the theorem.

The conditions for the composition of FIO's are open, they are still satisfied when the canonical relations are perturbed slightly. From (19) the following additional result follows.

Theorem 6.3 If the map $\Psi: \Lambda \rightarrow T^{*} X \backslash 0 \times E$ is $k$ to 1 at some imagepoint $(x, \xi, e)$, then $W\left(x, e, D_{x}\right)$ is in fact a sum of $k$ pseudodifferential operators in the following sense. When $H$ is taken with a background medium $c_{1}$ that is sufficiently small perturbation of $c_{0}$, then microlocally $W\left(x, e, D_{x}\right)$ changes into a sum of $k$ Fourier integral operators each with canonical relation that is a small perturbation of (18) at $(x, \xi, e)$.

Let us discuss the examples of section 4 . Denote by $\Lambda_{ \pm}$the subset of $\Lambda$ given by $\pm \tau>0$. In (10) and (11) the function $\mathbf{e}$ is chosen purposely 
so that the map $\Lambda_{ \pm} \rightarrow T^{*} X \backslash 0 \times E$ is a diffeomorphism to a subset of $T^{*} X \backslash 0 \times E$. Thus $H \circ F$ has a pseudodifferential part $W\left(x, e, D_{x}\right)$. The operator $W\left(x, e, D_{x}\right)$ is a sum of at most two contributions in the sense of Theorem 6.3 (each possible contribution corresponding to rays on one side of the reflector).

When $\mathbf{e}$ is given by (8), then away from caustics the map $\Psi$ is a local diffeomorphism, so that Theorem 6.1 applies. The map $\Psi$ may map several points in $\Lambda$ to the same value of $(x, \xi, e)$.

In the example (9) the map $\Lambda \rightarrow T^{*} X \backslash 0 \times E$ need not be a local diffeomorphism. We show this in the case of $n=2$ dimensions. In the presence of

multipathing it may happen that, away from caustics, $\frac{\partial r}{\partial \alpha}+\frac{\partial s}{\partial \beta}=0$, which, as one can show, implies that the map $\Psi$ is not a local diffeomorphism. Thus the conditions of Theorem 6.1 need not be satisfied.

\section{An example}

In this section we study an example for the scattering angle transform in 2 dimensions given by (7) and (10). We show that artifacts are present for a medium containing a horizontal reflector below a low velocity lens. Based on symmetry the presence of at least one artifact can be argued. By explicitly solving equations (14) we show that in fact a number of artifacts are present.

In Figure 2 as Gaussian low velocity lens is plotted centered at position $(0,1)$, as well as a horizontal reflector at depth 2 . The depth coordinate is 
plotted vertically downward. The medium above the reflector is given by

$$
c(x)=1-0.4 e^{-9(x-(0,1))^{2}} .
$$

For such a lens the rays will focus, and the wave fronts will develop singularities after passing through the lens. Some of the wavefronts and rays are given in Figure 3.

Consider the two raypaths drawn schematically in Figure 4. Source and receiver are located at $A=(0,0)$. Suppose a reflector is located at $B$. The raypath $\mathrm{ABA}$ leads to a singularity in the data at $s=0, r=0, t=T_{\mathrm{ABA}}$ (the traveltime along the depicted raypath $\mathrm{ABA}$ ). Because of the low velocity lens there is raypath ACA, depicted schematically in Figure 4, with the same traveltime. Because the medium is symmetric under reflection in the line $x_{1}=0$, there is an $\epsilon$ such that the fourth and fifth equations in (14) are also satisfied. Therefore a reflector at $B$ leads to an artifact in the image at $C$.

We study the solutions to (14) in more detail. The set of reflecting raypaths at the horizontal reflector can be parametrized by the horizontal coordinate of the reflection point $x_{1}$ and scattering angle $e$. The corresponding take off angles $\alpha, \beta$ can then be found and by solving the differential equations for the rays $\mathbf{r}(x, \alpha), \mathbf{s}(x, \beta), \phi_{r}(x, \alpha)+\phi_{s}(x, \beta)$ can be found.

The first three equation in (14) describe the isochron, the set of points $\bar{x}$ such that there are rays from $s=\mathbf{s}(x, \beta)$ and $r=\mathbf{r}(x, \alpha)$ to $\bar{x}$ with total traveltime $\phi_{r}(x, \alpha)+\phi_{s}(x, \beta)$. The solutions can be found without 


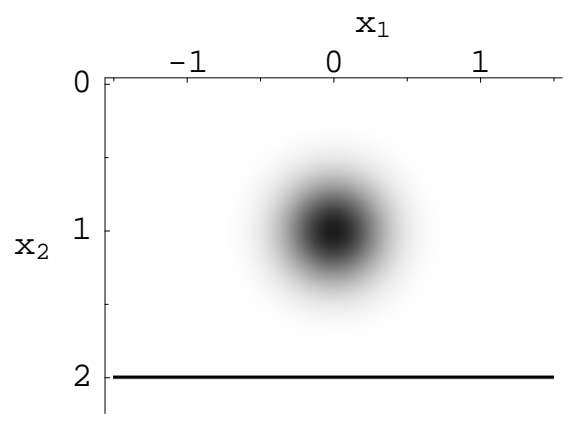

Figure 2: Densityplot of the medium and location of reflector.

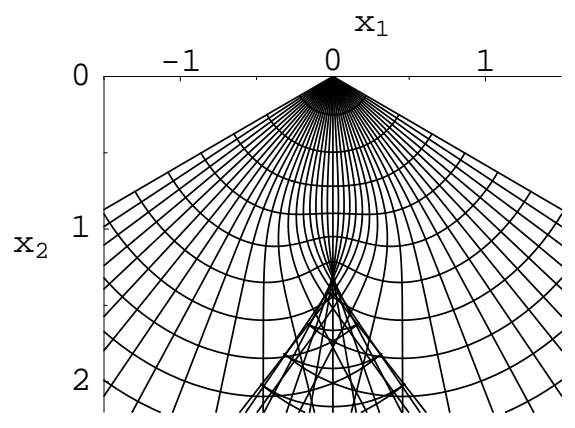

Figure 3: Some rays and wavefronts in the medium (20)

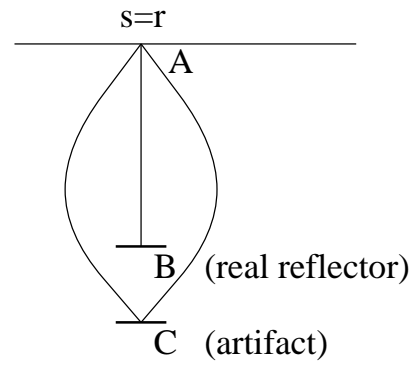

Figure 4: Schematic picture of raypaths leading to a solution of (14). 
computing the multivalued traveltime function by solving $\alpha_{s}, \alpha_{r}, t$ from

$$
\mathbf{x}\left(s, \alpha_{s}, \phi_{s}(x, \beta)+t\right)=\mathbf{x}\left(r, \alpha_{r}, \phi_{r}(x, \alpha)-t\right)
$$

and setting $\bar{x}=\mathbf{x}\left(s, \alpha_{s}, \phi_{s}(x, \beta)+t\right)$. The set of solutions $\left(\alpha_{s}, \alpha_{r}, t\right)$ of these equations is a smooth curve, except in exceptional points related to caustics or scattering over $\pi$. There exists $\epsilon$ such that the fourth and fifth equation of (14) are satisfied if only if

$$
\begin{aligned}
\left(\frac{\partial \mathbf{e}^{(i)}}{\partial s}(\bar{x}, r, s)\right)^{-1} & \left(\frac{\partial T^{(i)}}{\partial s}(\bar{x}, r, s)-\mathbf{p}_{s}(x, \beta)\right) \\
& =\left(\frac{\partial \mathbf{e}^{(i)}}{\partial r}(\bar{x}, r, s)\right)^{-1}\left(\frac{\partial T^{(i)}}{\partial r}(\bar{x}, r, s)-\mathbf{p}_{r}(x, \alpha)\right)
\end{aligned}
$$

where $s=\mathbf{s}(x, \beta), r=\mathbf{r}(x, \alpha)$. This equation can be used to find artifacts.

We have done these calculations for reflection point $x=(0,2)$, with scattering angle $e=\frac{\pi}{18}$ (10 degrees) and for $x=(0.3,2), e=\frac{2 \pi}{9}$ (40 degrees). This was done using the numerical differential equation solver and the root finder in the computer program Mathematica. The results are displayed in Figure 5. The fat solid line gives the raypath corresponding to the reflection. The other solid line gives the isochron related to this reflected raypair. In the subsurface for both source and receiver there is a region with three oneway traveltimes. Because in the first case the regions overlap, the isochron consists of 9 smooth pieces. In the second case the isochron consists of 5 smooth pieces. In addition we have drawn the various raypairs that lead to artifacts in the image (solutions of (21)) using dashed lines. The artifact will occur at position given by the intersection of the two rays on the isochron, and at scattering angle given by the angle between the two rays. 
Finally we wanted to make a section of the image volume, for given horizontal coordinate of the image. This means the horizontal component $\bar{x}_{1}$ of $\bar{x}$ is given in (14) is given. We plot the vertical component $\bar{x}_{2}$ of $\bar{x}$ and the angle parameter $e^{(i)}(\bar{x}, r, s)$ for all the solutions to (14). An examples of this is given in Figure 6. In addition the to the correct image that is displayed horizontally at depth 2 there are a number of artifacts. To achieve this we explicitly calculated an approximation to the multivalued traveltime function in Mathematica, using raytracing and interpolation. From the two dimensional set of reflected raypaths (parametrized by reflection point on the reflector and scattering angle), the ones leading to artifacts form a subset that is one dimensional when the equations satisfy the conditions of the implicit function theorem. For horizontal position 0.3 , somewhat to the side of the lens, a number of such one dimensional subsets could be found, and we plotted the corresponding artifact location. Here we omitted raypairs if the source or receiver coordinate was outside a certain interval, or if the value of a cutoff function we constructed was too small (recall that the amplitude in (7) goes to zero smoothly close to a caustic). We found that for horizontal position 0 there are a number of artifacts as well, but these are more difficult to plot because of degenerate solutions. 


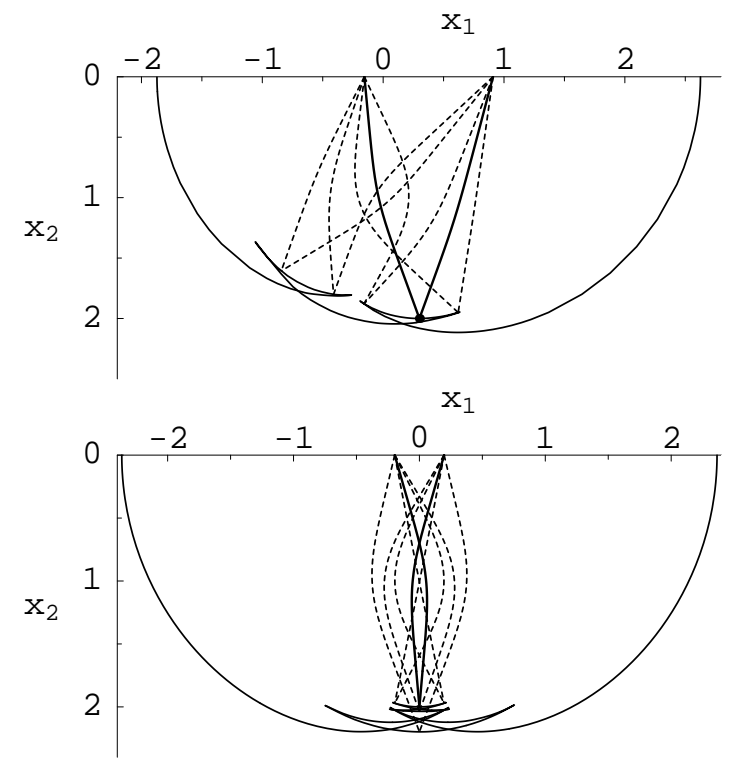

Figure 5: The isochron and rays leading to correct image and artifacts for a reflecting element at $x=(0.3,2), e=\frac{2}{9} \pi$, and for a reflecting element at $x=(0,2), e=\frac{\pi}{18}$.

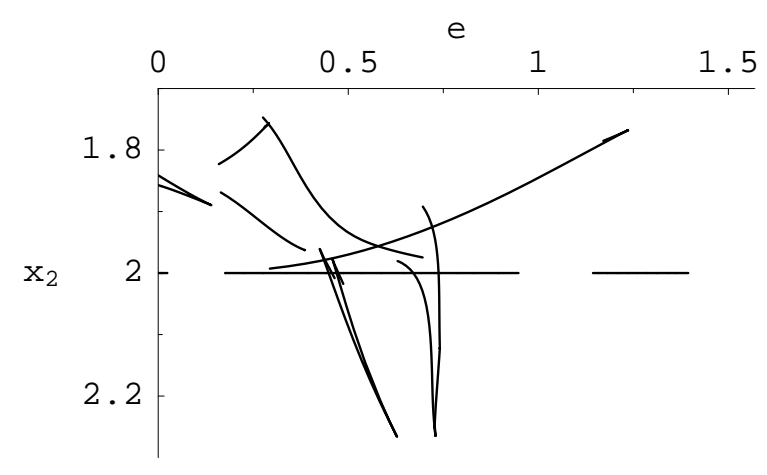

Figure 6: Positions of correct image and artifacts for the medium of Figure 2, for horizontal position $x_{1}=0.3$ 


\section{References}

[1] Beylkin, G. Imaging of discontinuities in the inverse scattering problem by inversion of a causal generalized Radon transform. J. Math. Phys. 1985, $26(1), 99-108$.

[2] Kroode, A. P. E. ten; Smit, D. J.; Verdel, A. R. A microlocal analysis of migration. Wave Motion 1998, 28, 149-172.

[3] Duistermaat, J. J. Fourier Integral Operators; Birkhäuser: Boston, 1996.

[4] Hörmander, L. The Analysis of Linear Partial Differential Operators; Springer-Verlag: Berlin, 1985; Vol. 4.

[5] François Treves. Introduction to Pseudodifferential and Fourier Integral Operators; Plenum Press: New York, 1980; Vol. 2.

[6] Stolk, C. C. Microlocal analysis of a seismic linearized inverse problem. Wave Motion 2000, 32, 267-290.

[7] Nolan, C. J.; Symes W. W. Global solution of a linearized inverse problem for the wave equation. Commun. Part. Diff. Eq. 1997, 22 (5-6), 919-952.

[8] Xu, S.; Chauris, H.; Lambaré, G.; Noble, M. Common angle migration: a strategy for imaging complex media. Preprint, École des Mines de Paris: Fontainebleau, France, 1999. 
[9] Brandsberg-Dahl, S.; Hoop, M. de; Ursin, B. Velocity analysis in the common scattering-angle/azimuth domain. In 69th Ann. Internat. Mtg.; Soc. Of Expl. Geophys.: Tulsa, Oklahoma, 1999; 1715-1718.

[10] Prucha, M.; Biondi, B.; Symes, W. Angle-domain common image gathers by wave-equation migration. In 69th Ann. Internat. Mtg.; Soc. Of Expl. Geophys.: Tulsa, Oklahoma, 1999; 824-827.

[11] Hoop, M. V. de; Symes, W. W.; Brandsberg-Dahl, S.; Ursin, B. Tomographic migration velocity analysis by differential semblance optimization with respect to scattering-angle/azimuth in anisotropic elastic media. Technical Report CWP-374, Center for Wave Phenomena, Colorado School of Mines: Golden, Colorado, 2001.

[12] Sava, P.; Biondi, B.; Fomel, S. Amplitude-preserved common image gathers by wave-equation migration. In 71th Ann. Internat. Mtg.; Soc. Of Expl. Geophys.: Tulsa, Oklahoma, 2001; to appear.

[13] Stolk, C. C. On the modeling and inversion of seismic data; Ph.D. thesis, Utrecht University: Utrecht, The Netherlands, 2000.

[14] Hörmander, L. The Analysis of Linear Partial Differential Operators, Springer-Verlag: Berlin, 1985; Vol. 3.

[15] Rakesh. A linearized inverse problem for the wave equation. Commun. Part. Diff. Eq. 1988, 13 (5), 573-601. 
[16] Hörmander, L. Linear partial differential operators. Academic Press Inc.: New York, 1963.

[17] Burridge, R.; Beylkin, G. On double integrals over spheres. Inverse Problems 1988, 4 (1), 1-10. 